\title{
Esofagitis Eosinofílica: Una entidad emergente
}

\author{
Eosinophilic Esophagitis, an emerging entity.
}

\author{
Constanza Beltrán $\mathrm{M}^{1}$, Raimundo García $\mathrm{M}^{2}$, Alberto Espino $\mathrm{E}^{3}$, Claudia Silva $\mathrm{A}^{4}$.
}

\begin{abstract}
RESUMEN
La esofagitis eosinofilica (EE) es una enfermedad primaria del esófago, previamente confundida con el reflujo gastroesofágico (RGE), cuyo conocimiento se ha desarrollado principalmente en la última década. Se define como la presencia de síntomas de disfunción esofágica (principalmente disfagia e impactación alimentaria), asociados a por lo menos una biopsia esofágica con más de 15 eosinófilos por campo de mayor aumento (CMA), y la exclusión de RGE. Su prevalencia va en aumento y afecta principalmente a niños y hombres jóvenes de raza blanca con historia previa de atopía. La EE sería causada por una reacción alérgica a ciertos alimentos y/o aeroalérgenos mediada por citoquinas y con cambios genéticos involucrados. La presentación clínica varía con la edad siendo la disfagia el síntoma más frecuente en todos los grupos etarios. El diagnóstico es clínico, endoscópico y anatomopatológico. Se requiere de una endoscopía digestiva alta (EDA) para evaluar hallazgos característicos y tomar biopsias para el estudio histológico. Los tratamientos actuales incluyen medidas dietéticas basadas en la eliminación de la exposición de alérgenos alimentarios y uso de corticoesteroides tópicos. El objetivo de esta revisión es analizar el estado actual de la definición de EE, historia, epidemiología, fisiopatología, diagnóstico y principalmente ayudar a mejorar su sospecha diagnóstica y manejo.
\end{abstract}

Palabras clave: Esofagitis eosinofílica, disfagia, atopía.

\begin{abstract}
Eosinophilic esophagitis (EE) is a primary disease of the esophagus, previously mistaken with gastroesophageal reflux disease (GERD). Its knowledge has developed over the last decade. EE is defined as the presence of esophageal dysfunction symptoms (mostly dysphagia and food impaction) associated to at least 1 esophageal biopsy with 15 or more eosinophils in 1 high-power field and absence of GERD. Its prevalence is rising, affecting principally white boys and young males with previous history of atopy. EE would be caused by an allergic reaction to certain food and aeroallergens mediated by citoquines with genetic changes involved. Clinical presentation varies with age being dysphagia the most common symptom in all age goups. The diagnosis is clinical, endoscopic and histopathologic. It requires an endoscopy to evaluate mucosal findings and to take the biopsies. Treatment includes elimination diets and topical steroids. The purpose of this review is to analyze the current state of the definition, history, epidemiology, fisiopathology and the diagnosis of EE, with an emphasis on improving its suspicion index and initial management.
\end{abstract}

Key words: Eosinophilic esophagitis, dysphagia, atopy.

\footnotetext{
Médico Otorrinolaringólogo, Departamento de Otorrinolaringología Pontificia Universidad Católica de Chile.

Interno de Medicina, Pontificia Universidad Católica de Chile.

Médico Gastroenterólogo, Departamento de Gastroenterología Pontificia Universidad Católica de Chile.

Médico Otorrinolaringólogo, Hospital Militar de Antofagasta.
} 


\section{INTRODUCCIÓN}

En condiciones normales, el esófago se encuentra libre de eosinófilos, por lo que el hallazgo de infiltración eosinofílica (IE) de la mucosa esofágica es considerado patológico. Se han descrito numerosas patologías en las que ocurre esta condición (Tabla 1), siendo la más frecuente el reflujo gastroesofágico (RGE), llegando incluso a considerarse la IE esofágica como reflejo de esta patología.

Sin embargo, en los últimos 10 a 15 años se ha observado un incremento de casos que se pensaba correspondían a RGE, pero que no respondían a la terapia con inhibidores de la bomba de protones (IBP) 0 a la cirugía antirreflujo. Estudios recientes han reconocido que estos casos pertenecían a una nueva patología independiente que afectaba tanto a niños como adultos y que ha emergido como una importante entidad epidemiológica. Se han utilizado diferentes términos para referirse a esta patología: esofagitis eosinofílica alérgica, esofagitis eosinofílica primaria, esofagitis eosinofílica idiopá-

Tabla 1. Patologías que presentan IE del esófago

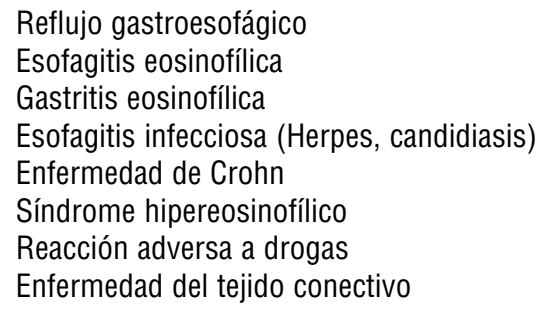

Adaptado de Furuta et al'1. tica; pero el término universalmente aceptado es el de esofagitis eosinofílica (EE).

\section{DEFINICIÓN}

En el año 2006, durante el consenso de recomendaciones para el diagnóstico y tratamiento de la $\mathrm{EE}$, la American Gastroenterology Association (AGA) definió la EE como una enfermedad primaria del esófago caracterizada por: (1) síntomas esofágicos y/o gastrointestinales altos, principalmente disfagia e impactación alimentaria en adultos, e intolerancia a los alimentos y síntomas de RGE en niños; (2) presencia de $\geq 15$ eosinófilos por campo de mayor aumento (CMA) en 10 más biopsias de mucosa esofágica; y (3) exclusión de RGE patológico evidenciado con un monitoreo de $\mathrm{pH}$ normal 0 por la falta de respuesta clínico-patológica a dosis altas de IBP1.

La definición implica la ausencia de IE de la mucosa gástrica y duodenal y el haber descartado razonablemente otras patologías que tengan características clínicas, endoscópicas o histológicas similares (Tabla 2) ${ }^{1}$.

\section{HISTORIA}

El primer reporte de IE del esófago corresponde al de Dobbins y cols en el año 1977 donde describieron el caso de un adulto con antecedentes de atopía que presentaba espasmo esofágico. En las biopsias se mostró una gastroenteritis eosinofílica (GE) con compromiso esofágico². Lo siguieron

Tabla 2. Características endoscópicas e histológicas de la EE

\begin{tabular}{|ll|}
\hline Endoscópicas & Histológicas \\
\hline Esófago de apariencia normal & Infiltración eosinofílica \\
Surcos lineales & Epitelio engrosado \\
Anillos concéntricos, esófago felino o traquealización & Papilas largas \\
Esófago de menor calibre, estenosis. & Lámina propia fibrótica \\
Exudados/placas blancas & Microabscesos \\
Fragilidad, desgarros lineales, mucosa de papel crêpe al paso endoscópico & \\
\hline
\end{tabular}

Adaptado de Gupte et al ${ }^{46}$ y Furuta et al ${ }^{1}$. 
otros reportes como el de Landres y cols donde se describió un caso de esofagitis eosinofílica en un hombre con acalasia, interpretándose como una posible variedad de GE que pudiese predisponer a un trastorno motor esofágico ${ }^{3}$. En los siguientes años se continuaron realizando asociaciones entre disfagia, alteraciones endoscópicas del esófago, IE y alergias alimentarias.

Desde el inicio de su descripción hubo discrepancias correspondientes al nombre de la enfermedad, los criterios diagnósticos y al tratamiento adecuado. Todo esto se vio potenciado por la falta de información y estudios por corresponder a una patología de reciente descubrimiento.

\section{EPIDEMIOLOGÍA}

La EE es una enfermedad de distribución mundial en que se han descrito casos en todos los continentes exceptuando África. Aunque se ha discutido respecto a variaciones geográficas de su prevalencia, la falta de estudios controlados en este momento hace imposible determinarlo. Si bien los primeros reportes apuntaban a que no habría susceptibilidades étnicas a la enfermedad, recientes estudios muestran una mayor incidencia en la población blanca ${ }^{4,5}$. No se ha estudiado su distribución socioeconómica.

Se estima que la prevalencia actual varía entre el $0,4 \%-1 \%$ en pacientes adultos y entre $0,04 \%$ $0,09 \%$ en niños con una incidencia anual de 1:100.000 en adultos y 1:10.000 en población pediátrica ${ }^{6-10}$. Desde la descripción de la enfermedad se ha reportado un continuo aumento en su prevalencia que no parece explicarse únicamente con un aumento en el reconocimiento de la patología. Este fenómeno podría deberse a que la EE es una patología crónica y reincidente, sin mortalidad asociada y con una incidencia estable, lo que lleva a un aumento de la prevalencia en el tiempo $0^{8,11}$.

Si bien los primeros reportes correspondían a población pediátrica, la evidencia actual indica que afecta tanto a niños como adultos. La mayoría de los adultos afectados son hombres jóvenes entre 20-50 años, con una edad promedio de 38 años. La edad promedio en los niños es de 8,6 años. Afecta principalmente a los hombres con una proporción 2-3:1 con respecto a las mujeres. Los síntomas reportados son similares entre ambos sexos ${ }^{1}$.
En Chile si bien existen reportes de la enfermedad no existen datos para conocer la prevalencia o incidencia ${ }^{12,13}$. Estos reportes muestran que las características epidemiológicas, clínicas y endoscópicas son similares a lo descrito mundialmente, lo que demostraría universalidad de la enfermedad.

\section{FISIOPATOLOGÍA}

La IE del esófago es tóxica para éste. Estudios in vitro han mostrado que el contenido de los gránulos eosinofílicos daña el epitelio ${ }^{14}$. La mucosa esofágica es dañada a través de mediadores inflamatorios como la proteína básica mayor eosinofílica, citoquinas y otras sustancias ${ }^{15,16}$. Una vez en la mucosa esofágica, los eosinófilos son capaces de perpetuar su presencia a través de la liberación de sustancias quimioatrayentes de eosinófilos como la interleuquina (IL)-3, IL-5 y el factor estimulante de colonias de macrófagos y granulocitos, produciendo un estado de inflamación crónica del esófago, lo que llevaría a su remodelamiento ${ }^{17,18}$.

Se ha establecido que la presencia de los eosinófilos en el esófago es patológica y con potencial de cronicidad. Lo que se desconoce actualmente es la causa de la primoinfiltración eosinofílica de la mucosa esofágica. Diversas teorías han surgido al respecto siendo la más aceptada actualmente la que postula que la etiopatogenia sería multifactorial, con componentes ambientales y genéticos, determinando una respuesta inmune alterada. Uno de los modelos propuestos consiste en que ciertos alimentos y aeroalérgenos gatillarían en el paciente una reacción de hipersensibilidad tipo I y tipo IV, causando una sobreexpresión de la eotaxina-3 y un aumento de la producción de citoquinas, principalmente IL-5 e IL-13, las cuales producirían un reclutamiento de eosinófilos a nivel esofágico ${ }^{18,19}$. El otro modelo atribuye al menos parte de la patogénesis de la EE al RGE que actuaría dañando directamente el epitelio esofágico, alterando su permeabilidad ${ }^{20}$.

De acuerdo a estos modelos descritos, los elementos involucrados en la fisiopatología de la EE son la atopía, gatillada por los alimentos y aeroalérgenos; la eotaxina-3 implicando un componente genético; las interleuquinas como media- 
doras de la inflamación y el RGE con un rol controversial.

Atopía. Existe una asociación clara entre la EE y la patología atópica demostrada porque la mayoría de Ios enfermos tienen antecedentes de alguna otra enfermedad alérgica ${ }^{1}$. En el caso de la EE algún antígeno gatillaría una reacción inflamatoria que Ilevaría a la IE del esófago. De acuerdo a las últimas investigaciones se cree que en la patogenia de la EE estarían involucrados los mecanismos de hipersensibilidad tipo I mediado por IgE y tipo IV mediado por Th2 que serían desencadenados por alimentos 0 aeroalérgenos, sin ser mutuamente excluyentes $^{21-23}$.

Alimentos. Existe evidencia que la exposición del esófago a ciertos alimentos gatillaría la reacción local en población pediátrica. Esto está respaldado por la mejoría clínico-patológica de ciertos pacientes al implementar un dieta elemental ${ }^{24,25} y$ de otros pacientes al identificar el antígeno y eliminarlo de la $\operatorname{dieta}^{26}$.

Aeroalérgenos. Hay estudios que sugieren que estos antígenos extraesofágicos pueden desencadenar una reacción en el esófago. Modelos experimentales en ratas muestran desarrollo de EE ante la exposición de antígenos en la tráquea, previa sensibilización cutánea o mucosa, fenómeno que no se repitió ante la exposición oral 0 intragástrica ${ }^{27,28}$. También hay evidencia de comportamiento estacional de la $\mathrm{EE}^{4,29}$.

Eotaxina-3. En el desarrollo embrionario temprano, Ios eosinófilos se establecen como residentes permanentes del tracto gastrointestinal bajo, proceso mediado por el péptido eotaxina ${ }^{30}$. Este péptido parece tener también un rol central en el reclutamiento eosinofílico mediado por antígenos, especialmente la eotaxina-3, que también jugaría un importante papel en la etipatogenia de la EE: se han demostrado niveles sanguíneos y esofágicos elevados y sobreexpresión genética de este péptido en pacientes con EE comparados con personas sanas, y modelos en ratas deficientes en el receptor de eotaxina-3 mostraron que estaban protegidas del desarrollo de $\mathrm{EE}^{22,31-33}$. Además se ha descrito la existencia de un polimorfismo de un solo nucleótido (SNP por sus siglas en inglés) en el gen de la eotaxina-3, lo que implicaría susceptibilidad genética a la $\mathrm{EE}^{32}$.

Genética. Además de las alteraciones en el gen de la eotaxina-3, la presencia de un componente genético en la etiología de la EE ha sido sugerida por estudios que muestran una agregación familiar, y el hecho que afecte mayoritariamente a hombres y a la raza blanca8,10,22.

Interleuquinas. Las IL juegan un rol fundamental en la patogénesis de la EE, especialmente las citoquinas de linfocitos Th2 con actividad estimulante de eosinófilos (IL-4, IL-5, IL-13). En biopsias esofágicas de pacientes con EE se ha observado un nivel elevado de éstas ${ }^{32}$. En modelos experimentales en ratas se ha establecido que las citoquinas Th2 son necesarias para el desarrollo de EE experimental; ratas knockout para IL-5 no desarrollaron IE esofágica y hubo ratas en que la EE también fue inducida con la sobreexpresión de las IL-5 e IL-13, citoquinas de los linfocitos Th2 ${ }^{27,28}$.

Reflujo gastroesofágico. Si bien existe una relación entre el RGE y la EE demostrado en que la prevalencia de RGE es mayor en pacientes con EE que en la población normal y sugerido por la resolución parcial de los síntomas de la EE con IBP en ciertos pacientes, todavía no se puede determinar la naturaleza de esta asociación ${ }^{20,34-36}$. Por una parte se cree que la EE contribuye o causa el RGE, esto porque los productos de secreción de los eosinófilos alteran la motilidad esofágica lo que favorece el RGE y dejan la mucosa más susceptible al daño ácido del jugo gástrico. Además la $E E$ causa cambios estructurales en el esófago (fibrosis, engrosamiento de la pared) que podrían afectar la función del esfínter esofágico inferior y la motilidad esofágica. Por otra parte, se piensa que el RGE contribuye 0 causa la EE, esto porque el daño ácido-péptico al epitelio esofágico altera su permeabilidad, exponiendo las capas profundas del epitelio a los antígenos que normalmente no entrarían en contacto, y además recluta células inmunes al epitelio lo que podría contribuir al desarrollo local de reacciones alérgicas ${ }^{20,34}$. 


\section{SÍNTOMAS Y SIGNOS (CLÍNICA)}

La presentación clínica de la EE varía con la edad, observándose una evolución cronológica de los síntomas presentados, aumentado su especificidad a medida que aumenta la edad de los pacientes (Figura 1). Este fenómeno podría reflejar una maduración en los procesos de señalización sensoriomotora, una progresión de la enfermedad u otra causa indeterminada todavía ${ }^{37}$.

El síntoma más común es la disfagia, que característicamente es crónica e intermitente sin interferir con la vida diaria ${ }^{38-42}$.

Niños. En esta población los principales síntomas son: intolerancia o rechazo alimentario y retraso del crecimiento en lactantes; vómitos, síntomas de RGE y dolor abdominal en escolares; disfagia e impactación alimentaria en adolescentes ${ }^{4,8,43-45}$.

Adultos. El principal síntoma es la disfagia intermitente y la impactación alimentaria, seguido de síntomas de RGE, dolor torácico. En un reporte se encontró que la EE era responsable del $50 \%$ de las impactaciones alimentarias esofágicas en consulta ambulatoria ${ }^{1,36}$.

Hay un retraso importante en el diagnóstico de la enfermedad en la población adulta, con un promedio de 4,5 años desde el inicio de los síntomas hasta el momento del diagnóstico. Esto se acentúa aún más en aquellos pacientes en que la enfermedad se manifiesta en forma progresiva y son capaces de adaptar sus hábitos alimentarios masticando la comida más lento y "lavando el alimento" con líquidos entre comidas, presentando síntomas más leves ${ }^{40,41,46}$.

Síntomas asociados. La mayoría de los pacientes (50\%-80\%), tanto adultos como niños, presentan alguna condición atópica: asma, rinosinusitis alérgica, urticaria, alergia alimentaria o eccema y se ha observado una variación estacional de los síntomas ${ }^{4,18,39,45}$.

Complicaciones. Las alteraciones estructurales esofágicas son las complicaciones más frecuentemente descritas. Éstas pueden ser desde cambios mínimos no detectables endoscópicamente con adelgazamiento de segmentos cortos o largos hasta estenosis significativa del lumen constituyendo una emergencia médica ${ }^{1}$. Se manifiestan como disfagia e impactación aguda de bolo alimentario. También se han descrito síndrome de Boerhaave (ruptura espontánea del esófago) y deficiencias nutricionales ${ }^{46-48}$.

\section{DIAGNÓSTICO}

La EE debe ser considerada siempre en: - Historia de impactación alimentaria,

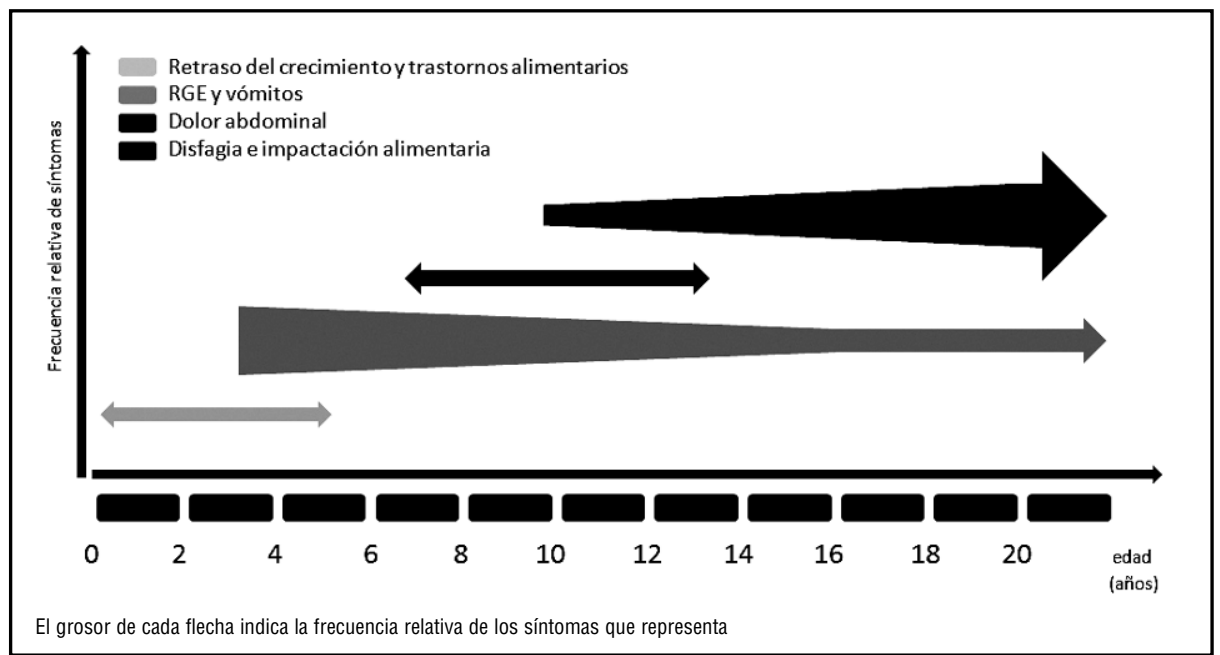

Figura 1. Síntomas de la EE por edad y frecuencia relativa. 
- Disfagia persistente en pacientes jóvenes especialmente si presentan historia de atopía, y

- RGE refractario a terapia.

El diagnóstico es clínico-patológico y requiere:

- Síntomas esofágicos,

- Biopsia esofágica con por lo menos un CMA con más de 15 eosinófilos.

- Biopsias normales en estómago y duodeno.

- Descartar RGE como causa de la eosinofilia: Estudio de $\mathrm{pH}$ esofágico normal 0 nuevas biopsias después de 6-8 semanas de tratamiento con IBP en altas dosis.

- Las características endoscópicas aunque no son categóricas, pueden sugerir fuertemente el diagnóstico.

\section{HERRAMIENTAS DIAGNÓSTICAS}

Endoscopía digestiva alta (EDA). Es el examen de elección en cualquier paciente que se sospeche EE, ya que permite realizar el diagnóstico y seguimiento.

Aspecto. Se han descrito numerosas alteraciones de la mucosa esofágica al examen endoscópico (Tabla 2 y Figura 2), ninguna patognomónica, que van desde el aspecto normal hasta estenosis severa del tracto esofágico. Si la EDA se realizó por otro motivo, la presencia de estos hallazgos debe hacer pensar en $\mathrm{EE}$, con mayor razón si existe además historia sugerente de síntomas de RGE. ${ }^{1}$
El aspecto endoscópico del esófago no es diagnóstico de $E E$, ya que hasta $30 \%$ de las endoscopías pueden parecer normales, aunque esto debería ir disminuyendo ya que actualmente existe por parte de los endoscopistas mayor atención a los cambios de la mucosa. Debido a la fragilidad de la mucosa descrita en la EE se debe tener cuidado durante la realización de la endoscopía ya que se han descrito desgarros y perforaciones ${ }^{48-50}$.

Biopsia. En un paciente con síntomas sugerentes de EE se deben tomar biopsias independientemente del aspecto de la mucosa esofágica. Igualmente si la EDA se realizó por otro motivo y se encuentran hallazgos sugerentes de EE con una historia orientadora. De acuerdo a la evidencia actual se recomienda intentar tomar 5 biopsias tanto del esófago proximal como distal, debido a que con una biopsia la sensibilidad para el diagnóstico de EE es de $55 \%$, aumentando hasta el $100 \%$ con las 5 biopsias $^{51}$. También se deben tomar biopsias del estómago y duodeno, con el objetivo de descartar otras enfermedades como GE, enfermedad inflamatoria intestinal, entre otras 1 .

Estudio de alérgenos. El diagnóstico de alimentos y aeroalérgenos, que incluye el prick test cutáneo para reacciones de hipersensibilidad tipo I mediadas por IgE y el patch test cutáneo para reacciones de hipersensibilidad tipo IV mediadas por Th2, ha

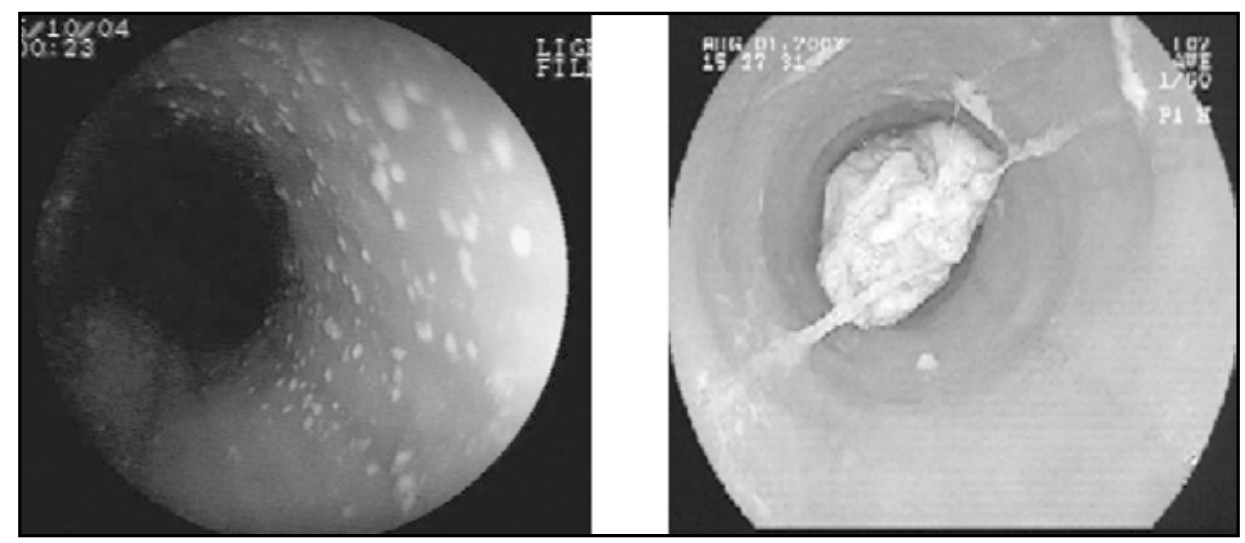

Figura 2. (A) Visión endoscópica de exudados blanquecinos que corresponden a microabscesos eosinofílicos. (B) Visión endoscópica de bolo alimenticio impactado en esófago (Fotos Depto. Gastroenterología PUC). 
demostrado utilidad para la identificación de las sustancias que causarían la EE en ciertos sujetos, permitiendo un tratamiento exitoso en la mayoría de los casos basado en los hallazgos de estos test ${ }^{21}$. Falta que su efectividad sea probada en más centros y en estudios con adultos.

El prick test implica aplicar extractos de alérgenos intracutáneamente y determinar la reactividad 15 minutos después, comparando con un control positivo de histamina y otro negativo con suero. El prick test es realizado con un stock estándar de carnes, granos, vegetales, frutas, soya, lácteos, maní y huevo. El patch test implica mezclar varios alimentos con suero salino isotónico y luego ponerlos en un parche en la espalda del paciente por 72 horas. A las 72 horas se evalúa al paciente y el hallazgo de eritema, pápulas o vesículas se leen como positivo.

Estudios no invasivos. Actualmente se está investigando en el desarrollo de marcadores no invasivos que podrían ser utilizados para el diagnóstico, clasificación de severidad, predicción de respuesta a ciertos tratamientos y seguimiento de la EE. Dentro de estos marcadores se encuentran los eosinófilos sanguíneos, el nivel de lgE sérico, CD23, eotaxinas y productos de la degranulación eosinofílica. Se espera que estos marcadores sean de gran utilidad en el futuro de la $\mathrm{EE}^{1,46}$.

\section{TRATAMIENTO}

\section{Dieta}

Existe una fuerte evidencia de que los alimentos contribuyen a la patogénesis de la EE en niños. Se ha demostrado claramente que la eliminación de antígenos alimentarios produce una mejoría tanto clínica como histológica de la EE en la mayoría de los pacientes ${ }^{52}$. Se han desarrollado 3 formas de eliminar estos antígenos alimentarios de la dieta: utilizar una fórmula elemental de alimentación que no contiene antígenos; la eliminación de alimentos específicos según la historia y pruebas de atopía; y eliminar los alimentos que se han demostrado son más alergénicos.

Dieta elemental. Consiste en reemplazar todos los alimentos sólidos por una fórmula elemental nutricionalmente completa en que la fuente proteica consiste exclusivamente en aminoácidos sintéticos. El uso de dietas elementales en niños y adolescentes ha mostrado ser efectiva en el $98 \%$ de los pacientes ${ }^{24,25}$. Se observó una resolución de los síntomas al día 8 en promedio, seguida de una resolución casi completa de la IE esofágica a las 45 semanas. Es mal tolerada por lo que en la mayoría de los pacientes debe ser administrada por sonda nasogástrica y puede llegar a ser muy costosa.

Dieta de eliminación específica. Consiste en evitar aquellos alimentos que se han identificado como alérgenos. Esto se puede realizar de acuerdo a la historia y test cutáneos ya sea el prick test 0 el patch test. Una vez identificados los alérgenos se debe administrar una alimentación libre de todos éstos, reintroduciendo a la dieta los alimentos identificados en forma progresiva cada 6 días y evaluando la sintomatología. Esta terapia ha mostrado ser efectiva en $78 \%$ de los pacientes utilizando ambos test cutáneos para aumentar el rango de alérgenos identificados ${ }^{21,26}$.

Dieta de eliminación estándar. Para disminuir los test realizados a los pacientes se realizaron estudios que demostraron que la eliminación de la dieta de los 6 alimentos que causan alergia más frecuentemente (lácteos, huevos, trigo, soya, maníes, pescados/mariscos) era un tratamiento efectivo en el $74 \%$ de los pacientes ${ }^{53}$.

La terapia basada en dieta debe ser considerada como una terapia efectiva en todos los niños diagnosticados con $\mathrm{EE}^{1}$. Al decidir el tipo de terapia no sólo se debe tener en cuenta su efectividad sino también el contexto biopsicosocial del niño y su familia. Además se debe contar con el apoyo de un nutricionista que asegure que la dieta sea la adecuada para los requerimientos del paciente. Es necesario realizar estudios antes de poder utilizar este tratamiento en adultos.

\section{Corticoides}

La inflamación eosinofílica se resuelve agudamente con el uso de corticoides sistémicos en varias enfermedades alérgicas incluyendo el asma y el eccema. Basándose en lo anterior se realizaron 
estudios con corticoides sistémicos que tuvieron buenos resultados pero luego se buscaron nuevas formas de administración (tópicas) para evitar los conocidos efectos adversos de la terapia corticoidea que, como se vería más adelante, debía ser crónica ${ }^{54}$.

Corticoides sistémicos. Hay diversos estudios que demostraron su efectividad en niños evidenciándose una mejoría clínico-patológica en la gran mayoría de los pacientes ${ }^{54-56}$. En los estudios más recientes se administró prednisona entre 1,5-2 mg/ $\mathrm{kg} / \mathrm{día}$ durante un mes. Se evidenció mejoría sintomática el día 8 en promedio, con una mejoría histológica evidenciada en la cuarta semana. En $40 \%$ de los niños se registraron efectos adversos corticoideos y en ambos estudios se demostró una recaída del $45 \%$ al controlarlos a los 6 ó 12 meses. La recaída ocurrió en promedio a la quinta semana de terminar la administración de prednisona. Si bien los corticoides sistémicos no parecen tener un rol en el tratamiento crónico de la EE debido a los considerables efectos adversos, especialmente en niños, sí parecen tener un rol importante en la terapia aguda en que es necesario lograr una remisión de la inflamación esofágica. Esto se produce en casos de disfagia aguda, riesgo de perforación esofágica por procedimientos, considerable pérdida de peso 0 falla de todas las otras terapias ${ }^{1}$.

Corticoides tópicos. Considerando las bases alérgicas de la EE y los efectos adversos de los corticoides sistémicos se comenzó a estudiar la administración de corticoides tópicos esofágicos. En diversos estudios se ha demostrado la efectividad tanto en niños como adultos con tasas de respuesta sintomática e histológica de 50\%-100\% a un plazo máximo de 4 meses $^{52}$. La duración de tratamiento típica es de 6-8 semanas. No se ha evaluado su uso como tratamiento crónico. No se han desarrollado corticoides específicos para la administración esofágica por lo que se utilizan corticoides inhalatorios sin aerocámara, los que se deben tragar, no aspirar, sin comer ni beber alimentos por media hora postadministración. La dosis recomendada de fluticasona es de 440-880 $\mathrm{mcg} / \mathrm{día}$ en niños y $880-1760 \mathrm{mcg} / \mathrm{día}$ en adultos, dividido en 2-4 dosis ${ }^{1,52}$.

Un estudio controlado comparó ambas vías de administración con resultados que sugirieron que la prednisona oral sería levemente más efectiva, pero la pequeña diferencia no se justificaría con la diferencia observada de efectos adversos: $40 \%$ oral (síntomas cushingoideos, hiperfagia, aumento de peso) v/s 15\% tópica (candidiasis esofágica) ${ }^{56}$.

\section{Inhibidores de la bomba de protones}

Anteriormente en este artículo se estableció la compleja relación de la EE con el RGE, por lo que utilizar IBP como tratamiento primario o coadyuvante se ha planteado en numerosos estudios. La recomendación actual es que los IBP no deberían ser considerados la terapia primaria de los pacientes con EE (como implica los criterios diagnósticos: si responde totalmente a terapia con IBP no corresponde a EE sino a RGE), pero pueden ser utilizados como coadyuvantes en ciertos pacientes ya que se ha observado una resolución parcial de los síntomas en algunos estudios ${ }^{1}$.

\section{Antagonistas de leucotrienos (ALT)}

Los leucotrienos son sustancias quimioatrayentes de los eosinófilos. En otras enfermedades atópicas como el asma se ha evidenciado la participación de los leucotrienos en su fisiopatología cumpliendo los ALT un rol en el tratamiento de la enfermedad ${ }^{57}$. Basándose en lo anterior es razonable pensar que los ALT podrían ser de utilidad en el tratamiento de la EE.

En un estudio con 8 pacientes adultos se mostró que con dosis elevadas del ALT Montelukast existía una remisión completa de los síntomas en 7 de éstos después de unas semanas de tratamiento. Luego se continuó el tratamiento en dosis menores por una mediana de 14 meses. Los síntomas recurrieron en 6 pacientes luego de disminuir la dosis o suspender el tratamiento. Histológicamente nunca disminuyó el conteo de eosinófilos de la mucosa esofágica lo que muestra que la remisión fue sólo sintomática sin mejorar la $\mathrm{IE}^{58}$. En otro estudio se compararon los niveles de cisteinil-leucotrienos en la mucosa esofágica de niños con EE y niños control, sin encontrarse diferencias, independiente del grado de inflamación. Esto sugiere que no habría un sustrato en donde podrían actuar los ALT ${ }^{59}$. Según la evidencia actual el uso de ALT no es una terapia recomendada en la $\mathrm{EE}^{1}$. 


\section{Biológica}

De acuerdo al conocimiento actual de la fisiopatología de la EE se han planteado terapias que actúen contra sustancias específicas identificadas dentro de la cascada inflamatoria, como es el caso del mepolizumab, un anticuerpo monoclonal humanizado contra la IL-5. Hay estudios pequeños que muestran resultados prometedores pero son necesarios mayores datos ${ }^{60,61}$. Actualmente se está realizando un estudio multicéntrico de mayor tamaño $0^{52}$.

\section{Tratamiento de las complicaciones}

Dilatación. La estenosis esofágica en diferentes grados es una complicación relativamente frecuente de la EE en niños y adultos ${ }^{51}$. Cuando se observa esta complicación la terapia médica puede que no sea suficiente y se tenga que recurrir a medios mecánicos para tratarla. La dilatación esofágica es útil en aquellos pacientes con estrechez esofágica secundaria a estenosis que causa impactación alimentaria. Sin embargo, debido al riesgo aumentado de desgarros mucosos y perforación por la fragilidad mucosa característica de la $\mathrm{EE}^{50}$, la recomendación es que, si es posible, se debe realizar una endoscopía diagnóstica con biopsia y tratamiento médico o dietético, previo a cualquier procedimiento de dilatación esofágica'.

\section{PRONÓSTICO}

La EE tiende a ser una enfermedad crónica con síntomas recidivantes, que no parece disminuir la expectativa de vida de quienes la padecen. Esta cronicidad hace relevante el pesquisar a tiempo patologías asociadas que se podrían desarrollar debido a las características de la enfermedad. En los niños puede producir retraso del crecimiento e intolerancia alimentaria. En los adultos las complicaciones más descritas son la impactación del bolo alimentario y la perforación, debido a la estenosis y adelgazamiento esofágico, respectivamente. $Y$ debido a que sin el tratamiento adecuado existe una condición de inflamación persistente en la mucosa esofágica se podría suponer que podría llevar al desarrollo de algún grado de metaplasia (esófago de Barrett, adenocarcinoma esofágico) en la zona afectada. Hasta la fecha ningún estudio demuestra que esto ocurra, pero hacen falta estudios prospectivos a largo plazo para poder descartar totalmente esta asociación'1.

\section{CONCLUSIONES}

La EE es una enfermedad emergente de distribución mundial de la que todavía se desconoce mucho. Afecta principalmente a niños y hombres jóvenes de raza blanca y la mayoría de las veces existe un componente atópico asociado. Se manifiesta generalmente como disfagia e impactación alimentaria y puede presentar complicaciones crónicas tanto en niños como en adultos.

Actualmente el diagnóstico requiere un alto índice de sospecha por parte del médico no especialista, quien debe estar atento tanto a los síntomas y signos gastrointestinales de la EE como a los síntomas acompañantes o sugerentes (retardo del crecimiento, rinitis alérgica, eccema, tos, asma, etc.). En todo paciente que se sospeche EE se debe solicitar una EDA indicando la sospecha diagnóstica para la adecuada visualización y toma de biopsias $y / 0$ ser derivado al especialista para una evaluación y manejo oportuno de la enfermedad.

Existen diversos tratamientos de la EE basados en los conocimientos actuales de su fisiopatología y se espera el desarrollo de nuevas alternativas en la medida en que se entienda más de esta enfermedad.

\section{BIBLIOGRAFÍA}

1. Furuta gt, liacouras CA, Collins mH et al. Eosinophilic esophagitis in children and adults: a systematic review and consensus recommendations for diagnosis and treatment. Gastroenterology 2007; 133: 1342-63.

2. Dobbins JW, Sheahan DG, Behar J. Eosinophilic gastroenteritis with esophageal involvement. Gastroenterology 1977; 72: 1312-6.

3. Landres RT, Kuster GG, Strum WB. Eosinophilic esophagitis in a patient with vigorous achalasia. Gastroenterology 1978; 74: 1298-301. 
4. Spergel JM, Brown-Whitehorn TF, Beausoleil YL, ET AL. 14 Years of Eosinophilic Esophagitis: Clinical Features and Prognosis. J Pediatr Gastroenterol Nutr 2009; 48: 30-6.

5. Assa'ad AH, Putnam PE, Collins MH, et al. Pediatric patients with eosinophilic esophagitis: an 8-year follow-up. J Allergy Clin Immunol 2007; 119: 731-8.

6. Ronkainen J, Talley NJ, Aro P, et al. Prevalence of eosinophilia and eosinophilic esophagitis in adults in the community: a random population based study. Gastroenterology 2006; 130: A575 (abstract).

7. Ronkainen J, Talley NJ, Aro P, et al. Prevalence of oesophageal eosinophils and eosinophilic oesophagitis in adults: the population-based Kalixanda study. Gut 2007; 56: 615-20.

8. Noel R, Putnman P, Rothemberg M. Eosinophilic esophagitis. N Engl J Med 2004; 351: 940-1.

9. BK Buckmeier, ME Rothenberg, MH Coluins. The Incidence and Prevalence of Eosinophilic Esophagitis. J Allergy Clin Immunol 2008; 121: S71 (abstract).

10. Straumann A, Simon H. Eosinophilic esophagitis: escalating epidemiology? J Allergy Clin Immunol 2004; 115: 418-9.

11. Vanderheyden AD, Petras Re, De Young BR, et al. Emerging eosinophilic (allergic) esophagitis: increased incidence or increased recognition? Arch Pathol Lab Med 2007; 131: 777-9.

12. Santander R, Schultz M, Roa I et al. Esofagitis Eosinofilica (EEo) Características clínicas y endoscópicas en Chile, Gastroenterol Latinoam 2008; 19: 269 (abstract).

13. Castro F, Barrueto K, González $C$ et al. Esofagitis eosinofílica en pacientes pediátricos y adultos. Gastroenterol Latinoam 2008; 19: 285 (abstract).

14. Gleich GJ, Frigas E, Loegering DA, Wassom DL, SteinmulleR D. Cytotoxic properties of the eosinophil major basic protein. J Immunol 1979; 123: 2925-7.

15. Mueller S, Aigner T, Neureiter D, Stolte M. Eosinophil infiltration and degranulation in esophageal mucosa from adult patients with eosinophilic esophagitis (EE). A retrospective comparative pathology biopsy study. J Clin Pathol 2006; 59(11): 1175-80.

16. Justinich CJ, Ricci A JR, Kalafus DA, Treem WR, Hyams JS, Kreutzer DL. Activated eosinophils in esophagitis in children: a transmission electron microscopic study. J Pediatr Gastroenterol Nutr 1997; 25: 194-8.

17. Desreumaux $P$, Bloget $F$, Seguy D, et al. Interleukin-3, granulocyte-macrophage colonystimulating factor, and interleukin-5 in eosinophilic gastroenteritis. Gastroenterology 1996; 110: 768.

18. Mishra A, Wang M, Pemmaraju VR, et al. Esophageal remodeling develops as a consequence of tissue specific IL-5-induced eosinophilia. Gastroenterology 2008; 134: 204-14.

19. Blanchard C, Mingler MK, Vicario M, et al. IL-13 involvement in eosinophilic esophagitis: transcriptome analysis and reversibility with glucocorticoids. J Allergy Clin Immunol 2007; 120: 1292-300.

20. Spechler SJ, Genta RM, Souza RF. Thoughts on the Complex Relationship Between Gastroesophageal Reflux Disease and Eosinophilic Esophagitis. Am J Gastroenterol 2007; 102: 1301-6.

21. Spergel JM, Beausoleil YL, Mascarenhas M, et al. The use of skin prick tests and patch tests to identify causative foods in eosinophilic esophagitis. J Allergy Clin Immunol 2002; 109: 363-8.

22. Blanchard C, Wang N, Rothenberg ME. Eosinophilic esophagitis: Pathogenesis, genetics, and therapy. J Allergy Clin Immunol 2006; 118: 1054-9.

23. Plaza-Martín am, Jiménez-Feijoo R, Andaluz C, et AL. Polysensitization to aeroallergens and food in eosinophilic esophagitis in a pediatric population. Allergol Immunopathol (Madr) 2007; 35: 35-7.

24. Kelly KJ, Lazenby AJ, Rowe PC, et al. Eosinophilic esophagitis attributed to gastroesophageal reflux: improvement with an amino acid-based formula. Gastroenterology 1995; 109: 1503-12.

25. Markowitz Je, Spergel JM, Ruchell E, Liacouras $C A$. Elemental diet is an effective treatment for eosinophilic esophagitis in children and adolescents. Am J Gastroenterol 2003; 98: 777-82.

26. Spergel JM, Andrew T, Brown-Whiteorn TF, et al. Treatment of eosinophilic esophagitis with specific food elimination diet directed by a 
combination of skin prick and patch tests. Ann Allergy Asthma Immunol 2005; 95: 336-43.

27. Mishra A, Hogan SP, Brandt EB, Rothenberg ME. IL-5 promotes eosinophil trafficking to the esophagus. J Immunol 2002; 168: 2464-9.

28. Mishra A, Hogan SP, Brandt EB, Rothenberg ME. An etiological role for aeroallergens and eosinophils in experimental esophagitis. J Clin Invest 2001; 107: 83-90.

29. Fogg MI, Ruchelli E, Spergel JM. Pollen and eosinophilic esophagitis. J Allergy Clin Immunol 2003; 112: 796-7.

30. Mishra, A, Hogan, SP, LeE, JJ, et al. Fundamental signals that regulate eosinophil homing to the gastrointestinal tract. J Clin Invest 1999; 103: 1719.

31. Hogan, SP, Mishra, A, Brandt, EB, et al. A critical role for eotaxin in experimental oral antigeninduced eosinophilic gastrointestinal allergy. Proc Natl Acad Sci USA 2000; 97: 6681.

32. Blanchard C, Wang N, Stringer KF, et al. Eotaxin3 and a uniquely conserved gene-expression profile in eosinophilic esophagitis. $J$ Clin Invest 2006; 116: 536-47.

33. Konikoff MR, Blanchard C, Kirby C, et al. Potential of blood eosinophils, eosinophilderived neurotoxin, and eotaxin-3 as biomarkers of eosinophilic esophagitis. Clin Gastroenterol Hepatol 2006; 4: 1328-36.

34. Remedios M, Campbell C, Jones DM, Kerlin P. Eosinophilic esophagitis in adults: clinical, endoscopic, histologic findings, and response to treatment with fluticasone propionate. Gastrointest Endosc 2006; 63: 3-12.

35. KaHRILAs PJ. Gastroesophageal Reflux Disease. N Engl J Med 2008; 359: 1700-7.

36. Desal tK, Stecevic V, Chang CH, et al. Association of eosinophilic inflammation with esophageal food impaction in adults. Gastrointest Endosc 2005; 61: 795-801.

37. Ferguson D, Foxx-Orenstein A. Eosinophilic esophagitis: an update. Diseases of the Esophagus 2007; 20: 2-8.

38. Potter JW, Saeian K, Staff D, et al. Eosinophilic esophagitis in adults: an emerging problem with unique esophageal features. Gastrointest Endosc 2004; 59: 355-61.

39. Fox VL, NuRko S, Furuta GT. Eosinophilic esophagitis: it's not just kids stuff. Gastrointest Endosc 2002; 56: 260-70.
40. Straumann A, Spichtin HP, Grize L, et al. Natural history of primary eosinophilic esophagitis: a follow-up of 30 adult patients for up to 11.5 years. Gastroenterology 2003; 125: 1660-9.

41. Croese J, Fairley SK, Masson JW, et al. Clinical and endoscopic features of eosinophilic esophagitis in adults. Gastrointest Endosc 2003; 58: 516-22.

42. Orenstein SR, Shalaby TM, Di Lorenzo C, Putnam PE, Sigurdsson L, KocochIS S. The spectrum of eosinophilic esophagitis beyond infancy: a clinical series of 30 children. Am J Gastrointest 2000; 95: 1422-30.

43. Teitelbaum J, Fox V, Twarog F, et al. Eosinophilic esophagitis in children: immunopathological analysis and response to fluticasone propionate. Gastroenterology 2002; 122: 1216-25.

44. Cheung KM, Oliver MR, Cameron DJ, et al. Esophageal eosinophilia in children with dysphagia. J Pediatr Gastroenterol Nutr 2003; 37: 498-503.

45. Liacouras CA, Spergel JM, Ruchelli E, et al. Eosinophilic esophagitis: a 10-year experience in 381 children. Clin Gastroenterol Hepatol 2005; 3: 1198-206.

46. Gupte AR, Draganov PV. Eosinophilic Esophagitis. World J Gastroenterol 2009; 15(1): 17-24

47. Straumann A, Bussmann C, Zuber M, Vannini S, Simon HU, Schoepfer A. Eosinophilic esophagitis: analysis of food impaction and perforation in 251 adolescent and adult patients. Clin Gastroenterol Hepatol 2008; 6: 598-600.

48. Cohen MS, Kaufman ab, Palazzo JP, Nevin D, Dimarino AJ JR, Cohen S. An audit of endoscopic complications in adult eosinophilic esophagitis. Clin Gastroenterol Hepatol 2007; 5: 1149-1153.

49. Fox VL. Eosinophilic esophagitis: endoscopic findings. Gastrointest Endosc Clin N Am 2008; 18: 45-57; viii.

50. Kaplan M, Mutlu EA, Jakate S, et al. Endoscopy in eosinophilic esophagitis: "feline" esophagus and perforation risk. Clin Gastroenterol Hepatol 2003; 1: 433-7.

51. Gonsalves N, Policarpio-Nicolas M, Zhang Q, Rao MS, Hirano I. Histopathologic variability and endoscopic correlates in adults with eosinophilic esophagitis. Gastrointest Endosc 2006; 64: 313-9. 
52. Вонm M, RICHTER JE. Treatment of EOsinophilic Esophagitis: Overview, Current Limitations, and Future Direction. Am J Gastroenterol 2008; 103: 2635-44.

53. Kagalwalla AF, Sentongo TA, Ritz S, et al. Effect of six-food elimination diet on clinical and histologic outcomes in eosinophilic esophagitis. Clin Gastroenterol Hepatol 2006; 4: 1097-102.

54. Liacouras C, Wenner W, Brown K, et al. Primary eosinophilic esophagitis in children: Successful treatment with oral corticosteroids. J Pediatr Gastroenterol Nutr 1998; 26: 380-5.

55. PICUS D, Frank PH. Eosinophilic esophagitis. AJR Am J Roentgenol 1981; 136: 1001-3.

56. Schaefer ET, Fitzgerald JF, Molleston JP, et al. Comparison of oral prednisone and topical fluticasone in the treatment of eosinophilic esophagitis: A randomized trial in children. Clin Gastroenterol Hepatol 2008; 6: 165-73.
57. Global strategy for asthma management and prevention (updated 2008): Global Initiative for Asthma (GINA). URL: http://www.ginasthma.org; 2008.

58. Attwood SE, Lewis CJ, Bronder CS, et al. Eosinophilic oesophagitis: a novel treatment using Montelukast. Gut 2003; 52: 181-5.

59. Gupta SK, Peters-Golden M, Fitzgerald JF, et al. Cysteinyl leukotriene levels in esophageal mucosal biopsies of children with eosinophilic inflammation: are they all the same? $A m \mathrm{~J}$ Gastroenterol 2006; 101: 1125-8.

60. Garrett JK, Jameson SC, Thomson B, et al. Antiinterleukin-5 (mepolizumab) therapy for hypereosinophilic syndromes. J Allergy Clin Immunol 2004; 113: 115-9.

61. Stein ML, Collins MH, Villanueva JM, et al. AntiIL-5 (mepolizumab) therapy for eosinophilic esophagitis. J Allergy Clin Immunol 2006; 118: 1312-9. 